\title{
A Wide-Area SVC Controller Design for Inter-Area Oscillation Damping in WECC based on a Structured Dynamic Equivalent Model
}

\author{
Matthew Weiss ${ }^{\mathrm{a}}$, Backer N. Abu-Jaradeh ${ }^{\mathrm{b}}$, Aranya Chakrabortty ${ }^{\mathrm{a}, 1, *}$, \\ Arash Jamehbozorg ${ }^{\mathrm{c}}$, Farrokh Habibi-Ashrafi ${ }^{\mathrm{b}}$, Armando Salazar ${ }^{\mathrm{b}}$ \\ ${ }^{a}$ North Carolina State University, Raleigh, NC 27695, USA. \\ ${ }^{b}$ Southern California Edison, Westminster, CA 92705, USA. \\ ${ }^{c}$ California State University, Los Angeles, CA 90032, USA.
}

\begin{abstract}
In our recent work [1] we constructed a reduced-order model of the Western Electricity Coordinating Council (WECC) power system using mathematically derived parameters from real Synchrophasor data. These parameters include inter and intra-area impedances, inertias, and damping factors for aggregate synchronous generators representing five geographical, and yet coherent, areas of WECC. In this paper we use this reduced-order model as a tool to design a supplementary controller for a Static VAr Compensator (SVC), located at the terminal bus of one of the aggregate generators. Widearea feedback consisting of phase angle and frequency measurements from Phasor Measurement Units (PMUs) in the other areas is used to design this controller. The objective is to damp the inter-machine oscillation modes of the reduced-order model, which in the full-order system corresponds to inter-area oscillations. The controller input is chosen via statistical variance analysis, and its parameters are tuned to improve the damping factors of the slow modes. The model is implemented in a real-time digital simulator, and validated using a wide range of disturbance scenarios. The closed-loop system is observed to be highly robust to all of these disturbances as well as the

${ }^{*}$ Corresponding author

Email addresses: mdweiss@ncsu.edu (Matthew Weiss),

Backer.Abu-Jaradeh@sce.com (Backer N. Abu-Jaradeh), achakra2@ncsu.edu (Aranya Chakrabortty ), ajamehb@calstatela.edu (Arash Jamehbozorg),

Farrokh.Habibiashrafi@sce.com (Farrokh Habibi-Ashrafi), Armando.Salazar@sce.com (Armando Salazar)

${ }^{1}$ This research is partially supported by grant DE-OE0000654 from the US Department of Energy.
\end{abstract}


choice of operating points. Detailed experimental analyses of the capacity of the SVC to satisfy the damping specifications of supplementary control are also presented via multiple contingencies. The results are promising in aiding damping of inter-area modes in WECC, especially at a time of increasing penetration of wind and other renewable resources.

Keywords: Wide-area control, Synchrophasors, Static VAr Compensator (SVC), Dynamic equivalencing, Inter-area oscillations, SVC Droop

\section{Introduction}

The Western Electricity Coordinating Council (WECC) is responsible for coordinating and promoting bulk electric power system reliability in the western part of North America. The geographic size and diversity of the WECC leads to a special network topology with separate and well-defined generation and load centers, with long tie lines connecting these various regions. This results in coherency between the generators [2], and therefore, a well-defined time-scale separation of the slow and fast electro-mechanical modes of oscillation within the $500 \mathrm{kV}$ network of WECC [3]. The slow oscillations, typically referred to as inter-area oscillations, are well-studied for the traditional operating conditions of the WECC. However, with gradual expansion in the transmission infrastructure as well as tremendous penetration of renewable power including wind and solar photovoltaic in the west coast over the next decade, several dynamical properties of the WECC will change significantly, and so will the characteristics of the interarea oscillations and their stability margins. Such projected changes are neither well-understood from an analytical perspective nor well-established from experiments. Our goal in this paper is to - (1) design a wide-area FACTS controller that will integrate real-time phasor measurements of voltage, phase angle and frequencies from different points in the WECC model in order to damp these inter-area oscillations over a wide range of operating conditions including unforeseen contingencies and intermittent renewable generation, and (2) thereafter, validate those observations using an RTDS ${ }^{2}$ based emulation framework. To achieve these goal, we use a reduced-order dynamic equivalent model of the 5 -area WECC system that was recently developed in our paper [1] using Synchrophasors. We start from this reduced-order swing model of the WECC, and design a wide-area supplementary controller for

\footnotetext{
${ }^{2}$ Real-Time Digital Simulator
} 


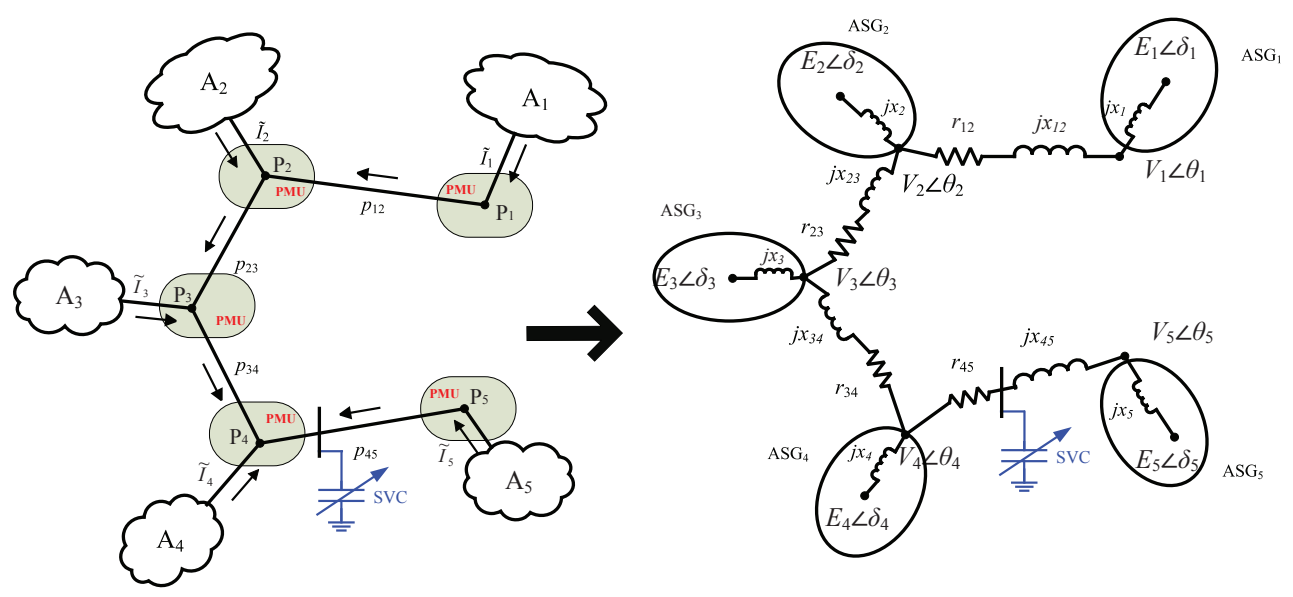

Figure 1: Electrical topology of WECC's $500 \mathrm{kV}$ network

a Static VAr Compensator (SVC) sited between two areas. Following the Static VAr System installed in between southern California and Arizona regions of WECC [7], we choose the SVC location at an intermediate bus between areas 4 and 5, as shown in Figure 1. The objective is to damp the oscillation modes between all the aggregate machines, which in the actual system will correspond to inter-area oscillations, i.e., oscillations between all the areas. The interesting aspect of this design is that although it is based on a reduced-order model, the designed controller can be easily implemented in the actual WECC system since the pilot buses retain their identity. Note, however, that the structure of the model mandates the controller to be a in shunt configuration. For example, one will not be able to use this model to design a series controller such as a Thyristor Controlled Series Compensator (TCSC) along a transmission line connecting two areas [5, 6], simply because of the fact that the tie-lines retained in the model are equivalent tielines that do not exist in the actual system. Similarly, conventional damping controllers such as Power System Stabilizers (PSS) are also not permissible for this model since all the ASG's are aggregate generators.

The basic approach for the control design presented here is twofold. First, a nominal PI controller is designed using local output feedback, and the gains are tuned for optimal closed-loop damping of the inter-area power flows. Thereafter, a supplementary controller is designed using remote feedback of voltages and phase angles from other pilot buses, thereby forming a wide-area control loop. Several studies have been done in recent past on FACTS/SVC design for oscillation damping [9]. Majority of these de- 
signs, however, need precise information about all the intra-area network and machine parameters surrounding the FACTS device. Our controller, in contrast, only needs aggregate model information, and PMU data feedback from the pilot buses, and therefore is much easier to design, and simpler to implement, once the reduced-order model is estimated accurately.

\section{Description of the 5-Area WECC Model}

As mentioned in the introduction, the WECC system is divided into five non-overlapping coherent areas, connected in a linear topology through long $500 \mathrm{kV}$ transmission lines following the example cited in [3]. These five areas are represented by five aggregated synchronous generators (ASG) with the interconnecting $500 \mathrm{kV}$ lines between any two areas reduced to a single equivalent transmission line, as shown in Figure 1. The lines connect five real-world sub-stations, referred to as pilot buses that are selected from each area based on two criteria - (1) the sub-station must have a PMU installed at its location, and (2) all generators within that area must lie behind this sub-station. The voltage phasor $V_{i} \angle \theta_{i}(t)$ is known at any pilot bus $i$ owing to availability of PMU data at that bus over time $t$. Furthermore, the current $I_{i} \angle \alpha_{i}(t)$ injected at pilot bus $i$ can be calculated from the difference in line currents flowing in and out of that pilot bus, known from PMU data:

$$
\tilde{I}_{i}(t)=\tilde{I}_{i k}(t)-\tilde{I}_{j i}(t), \quad j, k \in \mathcal{N}_{i},
$$

where $\mathcal{N}_{i}$ is the neighboring pilot bus set for $i$. The pilot bus of a particular area also acts as the terminal bus for the ASG in that area. Looking from the pilot bus into the area, this generator is modeled as a Thevenin voltage source with internal EMF $E_{i} \angle \delta_{i}$ and Thevenin reactance $j x_{i}$. Due to non-identifiability, it is not possible to model it as an impedance of $r_{i}+j x_{i}$ (for details please see [1]). Each ASG is modeled as a second-order damped oscillator described by the swing equations. Since these ASGs are fictitious generators, their model parameters are not known, and need to be identified using PMU measurements of voltage and phase angles measured at the corresponding pilot bus. Thus, the parameter identification for the fivearea model is a three-step process: (1) Identification of inter-area tie-line impedance $\left(r_{i j}+j x_{i j}\right)$ connecting pilot bus $i$ to pilot bus $j$, (2) Identification of Thevenin reactance $j x_{i}$ of ASG $i$, and (3) Identification of inertia $M_{i}$ and damping $D_{i}$ of ASG $i$. Since the aggregate generators are obtained by collapsing the collections of actual coherent generators, the non-coherent modes or local modes must be removed from the PMU measurements before they can be used to identify the above three quantities for each ASG. 
In other words, the raw PMU data contains both fast local modes as well as slow inter-area modes, and must be passed through a band-pass filter to remove the fast modes that do not correspond to the inter-machine oscillations in the reduced-order model. However, since such filtering typically adds a phase shift to the slow modes, distorting the data, an alternative approach is to use time-domain decomposition methods, also typically referred to as subspace identification or modal decomposition. Commonly used modal decomposition methods include Prony and Eigensystem Realization Algorithm (ERA) [8], which are time-domain based curve-fitting techniques that can be used to determine frequency, amplitude, phase and damping components of the 'equivalent' PMU measurement from the pilot buses.

\section{SVC Nominal Controller Tuning}

A Static Var System (SVS) is defined as a combination of discretely and continuously switched Var sources that are operated in a coordinated fashion by an automated control system. Following the WECC SVC report in [7] we assume the SVS model for our control design to be comprised of a thyristor based SVC, coupled with coordinated mechanically switched shunts (MSSs). It is assumed that at least one thyristor controlled reactor (TCR) branch exists. Thus, for the purpose of positive sequence simulations, the SVC can be modeled as a smoothly and continuously controllable susceptance throughout its entire operating range. We consider a SVC located between areas 4 and 5 that includes a 117 MVAR variable inductive reactance, and two 91 MVAR switchable capacitor banks. A droop of $4 \%$ is necessary to allow the SVC to operate within limits during faults of desired excitation capabilities. Figure 2 shows a comparison of the transient responses of all four inter-area phase angle differences between adjacent pilot bus pairs. The datasets plotted here include the baseline WECC model when no SVC is operational against the case in which this SVC is brought online half way between areas 4 and 5 . The baseline WECC model also includes 500 Megawatts of wind penetration located at area 4 . The transient response is created by a 4-cycle line-to-ground fault in area 3 .

The SVC alone is responsible for improving the transient response of the inter-area power flows even without adding supplementary wide-area control. We first design a nominal controller for the SVC using a simple PI controller whose input is a per-unit voltage measurement of the local bus where the SVC unit is located. This voltage input signal is filtered to eliminate fast modes via a bandpass filter (retaining only modes between $0.1 \mathrm{~Hz}$ and $2 \mathrm{~Hz}$ ), and the SVC droop is compensated for before the signal reaches 
the input of the PI controller. The output of this PI controller is responsible for controlling the reactive elements in the SVC. The two PI controller constants, $k_{p}$ and $k_{i}$, are tuned to appropriate values to optimize the SVC performance using Zeigler-Nichols tuning method. The nominal values are found to be $k_{p}=5.18$ and $k_{i}=0.167$. Upon installing this SVC with its nominal local controller into the WECC model implemented in real-time digital simulator (RTDS) the slow-mode damping of the electro-mechanical oscillations is noticeably improved, as shown in Figure 2. However, significantly more improvement using wide-area control, i.e., by feeding back signals from the remote pilot buses, is possible. This motivates us to design an additional supplementary controller that works in conjunction with the local nominal controller.
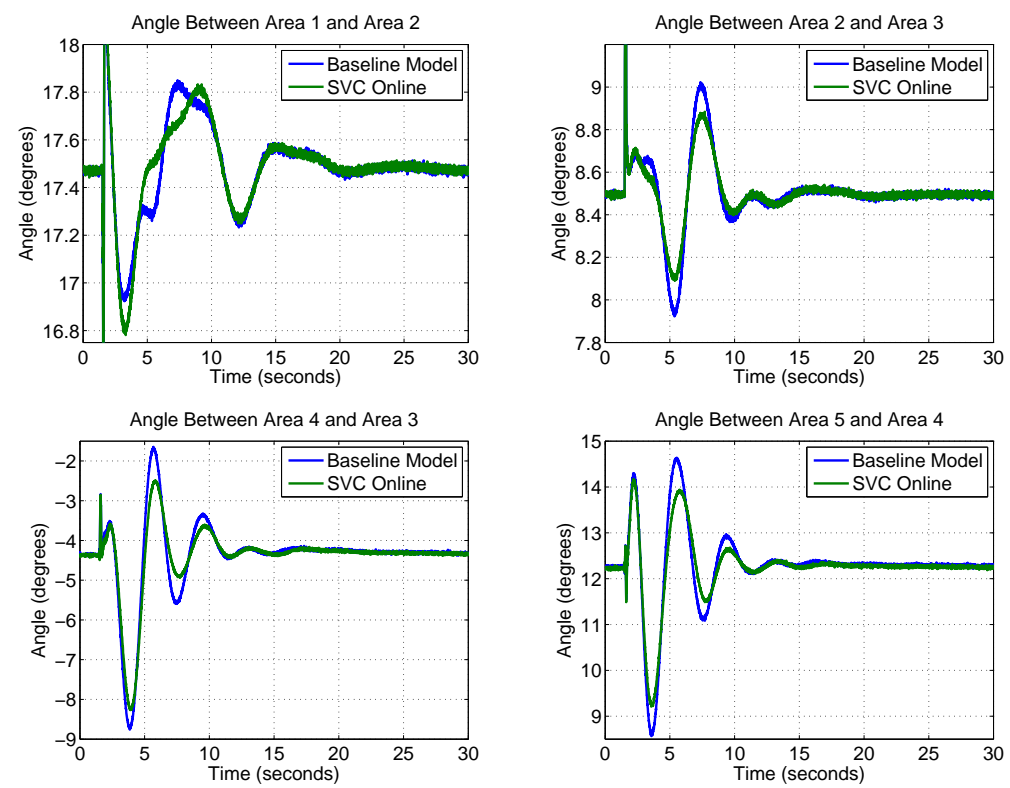

Figure 2: Improvement of dynamic response with SVC and nominal control

\section{Selection of Supplementary Control Input}

The supplementary controller for the SVC requires an additional input signal besides the standard voltage feedback from the local pilot bus. The viable choices for this feedback variable are phase angles, voltages, and frequency of the remote pilot buses, or the power flow across any of the equivalent tie-lines. The reduced-order model in our example is validated 
by matching the phase angles at the pilot buses with those obtained from real PMU data, as a result of which the phase at these buses are very accurate representations of the actual measured phases. Therefore, we choose phase angle as the feedback variable. Figure 3 shows the supplementary controller. The input to this controller for both branches is selected by the process shown in Subsections 4.2-4.3. The output of this controller is added to the voltage measurement of the local bus in per unit, and the resulting sum is applied to the nominal PI controller.

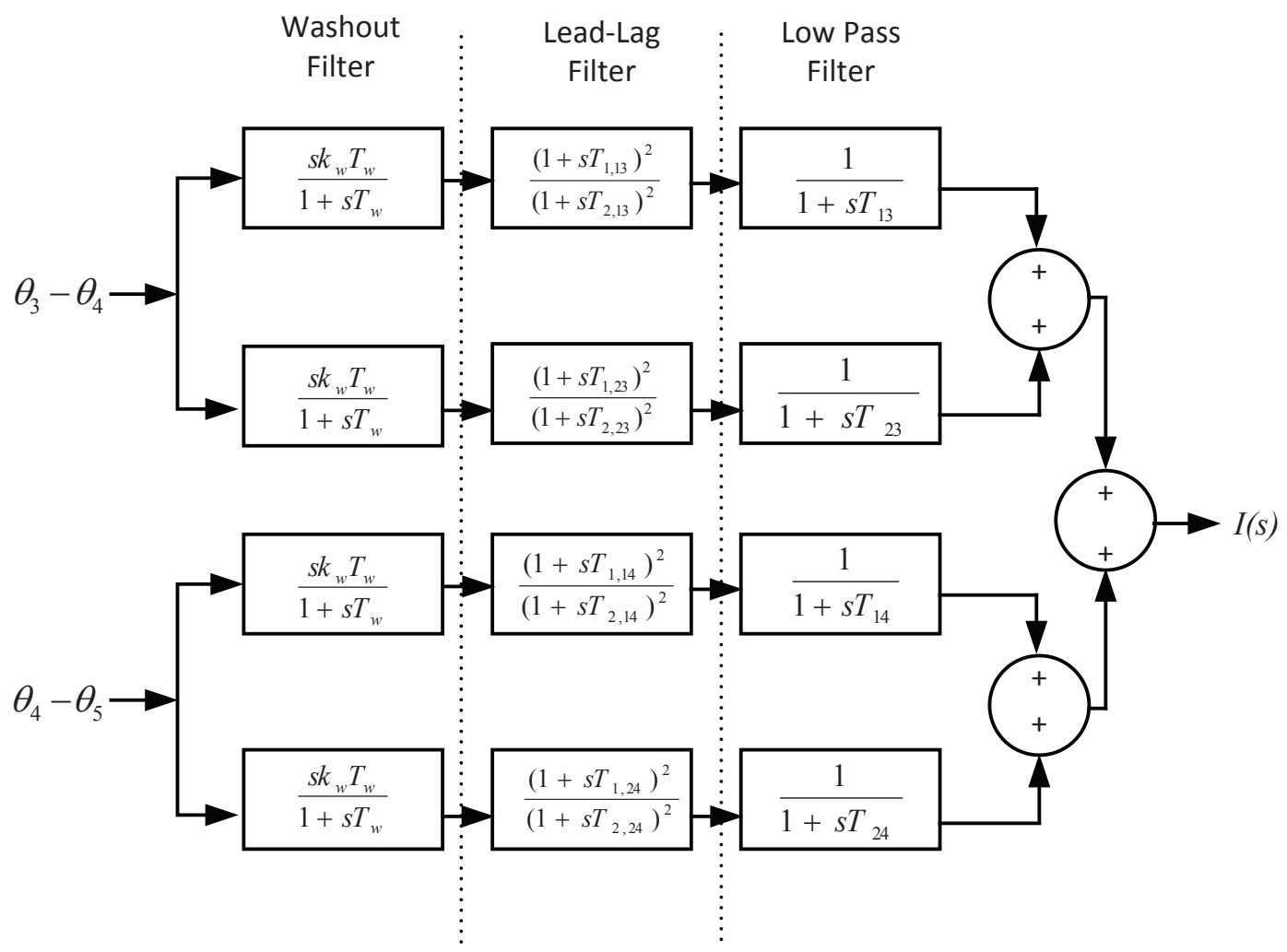

Figure 3: Supplementary Controller Structure

\subsection{Choice of Control Metric}

In order to measure the improvements made by a controller on the transient response of the power system, a metric needs to be defined. The WECC has five aggregate machines and, therefore, four slow oscillation modes. However, every pilot bus phase angle may not exhibit the same dominant 
contribution of all the slow modes. Therefore, again, a modal decomposition algorithm based on subspace identification methods such Eigensystem Realization Algorithm (ERA) is used to evaluate the residues or participation of every slow mode on the pilot bus phase angles using simulated fault response data. The slow mode frequencies, residues, and damping values are estimated using ERA, and the angles with highest residues of the slow modes are chosen as suitable candidates for observed outputs on which the impact of the SVC can be evaluated.

\subsection{Selection of an Input Signal}

We next consider the selection of the optimal feedback variable for the supplementary controller. This decision is made using the variable which is most robust to changes in the pre-disturbance equilibrium or steady-state operating point of the system. An impulse input to the SVC controller is applied, and the resulting transient response of the inter-area phase angles is decomposed using ERA. This process is repeated for many different randomized operating points, for example by varying the model parameters of the five-machine model. These parameters include the load reference of the governor of each machine, and the amount of wind penetration at area 4 . By randomizing these parameters, a randomized profile of the power flows across the equivalent transmission lines, and thus a randomized profile of the steady-state phase angles across these lines is created using a Gaussian distribution. While not identical, the phase angle variations in the model due to these randomized parameters distribute across all four phase angles fairly similarly. The model is then excited using an impulse input, and the resulting transient responses of the four inter-area phase angle differences are collected and decomposed by ERA. Figure 4 plots several transient responses against one another when the power flows are in various randomly generated steady-states. Each trace on the plot represents the transient response corresponding to a random operating point. Each phase angle was found to exhibit two dominant slow-mode frequencies that varied in residue as the steady-state operating point is varied. Often there were more modes of oscillation with residue less than that of the dominant poles by several orders of magnitude. These poles are neglected due to their insignificance in shaping the transient responses, and only the two most important pole pairs were retained. Mode 1 is the slowest oscillation mode, and Mode 2 is the relatively faster mode. 

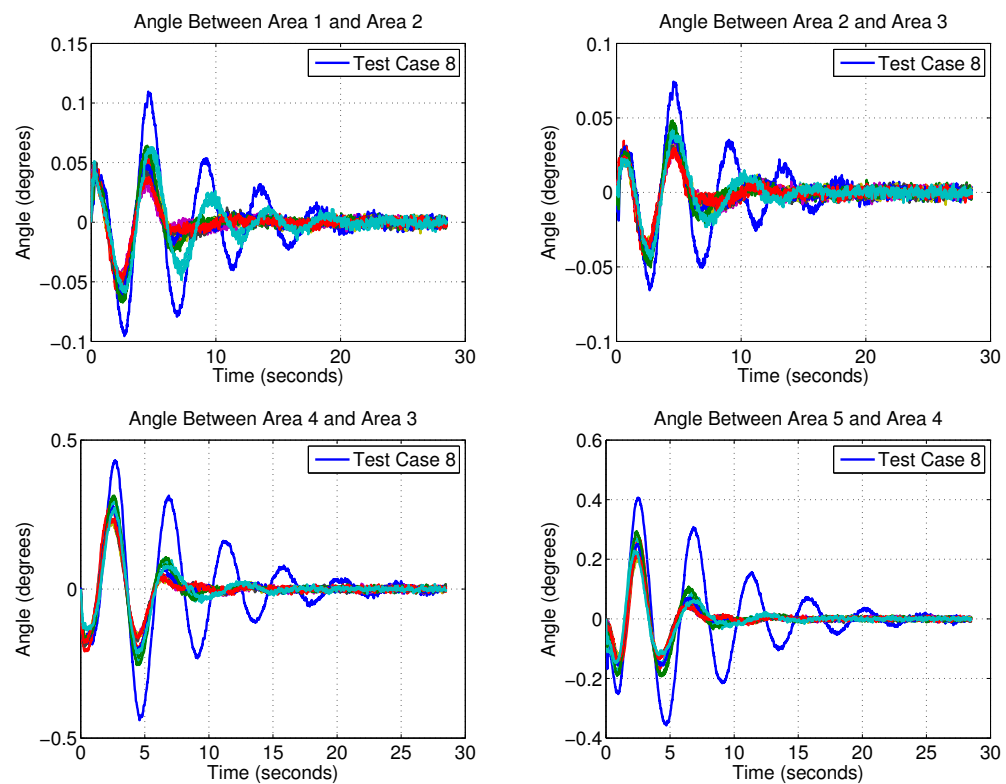

Figure 4: Phase Angle Response from Randomized Tests

\subsection{Variance in the Residues of Slow Modes}

For convenience of notations, from this point onward we will often use the phrase 'phase angle' to denote phase angle difference across the transfer paths in the reduced-order model. For example, phase angle 1 refers to the angle difference between pilot buses 1 and 2, phase angle 2 refers to the difference between pilot buses 2 and 3 , and so on. The variance of each mode $i$ on each phase angle $j$ across all $k$ test cases was calculated off the residues as:

$$
\begin{gathered}
P_{i j k}=\frac{R_{i j k}}{s^{2}+2 d_{i j k} \omega_{i j k} s+\omega_{i j k}^{2}}=\frac{A_{i j k}}{\left(s+C_{i j k}\right)}+\frac{A_{i j k}^{*}}{\left(s+C_{i j k}^{*}\right)} \\
V_{i j}=\operatorname{Variance}\left(A_{i j 1} \ldots A_{i j k}\right)
\end{gathered}
$$

Table 1: Variance of Phase Angle Differences

\begin{tabular}{|c|r|c|c|c|}
\hline Mode & Phase 1 & Phase 2 & Phase 3 & Phase 4 \\
\hline 1 & 0.7374 & 0.0486 & 0.0427 & 0.0401 \\
2 & 0.7376 & 0.0458 & 0.0583 & 0.3468 \\
\hline
\end{tabular}


where estimation of the residue $R_{i j k}$, damping $d_{i j k}$, and angular frequency $\omega_{i j k}$ of mode $i$, phase angle $j$ follow from ERA. In fact, ERA yields these values as conjugate pole pairs, which is thereby transformed to a pole-residue form shown in (2) for computation of the controller parameters. One simulation case, shown in Figure 4 , is significantly less damped than the rest of the cases. Table 1 displays the variances with this outlier case omitted.

Table 1 shows that three phase angles were relatively insensitive to changes in the operating point, whereas phase angle 1 was fairly sensitive. When the outlier is not omitted, however, phase angle 2 demonstrates a significant amount of variation, and makes it less attractive as a feedback variable, leaving phases 3 and 4 as reasonable choices. Since the SVC is actually located along the transmission line where phase angle 4 is measured (i.e. the angle difference between pilot buses 4 and 5), phase angle 3 is chosen first in the interest of feeding back a variable that is closest to the SVC site. Implementation of the controller using both phase angles 3 and 4 is considered as well. Note that the latter means that angles from all three pilot buses 3, 4 and 5 are fed back to the SVC.

\section{Parameter Calculations for SVC Supplementary Controller}

We next describe the construction of the controller transfer functions for our SVC model as shown in Figure 3. The typical approach is to develop multiple cascades of controllers, each responsible for filtering specific modal information from the feedback signal to actuate the required damping torque [10]. For simplicity, we use a 3-stage controller as shown in Figure 3 consisting of two parallel paths, one for each phase angle feedback. First, modal decomposition of phase angles 3 and 4 is performed using ERA. The results for the two dominant modes are shown in Table 2 ,

Table 2: Phase 3 and 4 Modal Data

\begin{tabular}{|c|c|c|c|c|}
\hline $\begin{array}{c}\text { Angle } \\
j\end{array}$ & $\begin{array}{c}\text { Mode } \\
i\end{array}$ & $\begin{array}{c}\text { Frequency } \\
\omega_{i j 0}(\mathrm{rad} / \mathrm{s})\end{array}$ & $\begin{array}{c}\text { Residue } \\
R_{i j 0}\end{array}$ & $\begin{array}{c}\text { Damping } \\
d_{i j 0}\end{array}$ \\
\hline $3-4$ & 1 & 1.2478 & 45.5570 & 0.2719 \\
$3-4$ & 2 & 1.9131 & 42.2355 & 0.2099 \\
$4-5$ & 1 & 1.2784 & 41.0623 & 0.2763 \\
$4-5$ & 2 & 1.7526 & 64.0806 & 0.2813 \\
\hline
\end{tabular}

Based on these estimates, the controllers in each parallel path are com- 
puted as follows:

- Define

$$
\begin{aligned}
\phi_{i j} & =180^{\circ}-\angle\left(A_{i j 0}\right), \quad \alpha_{i j}=\frac{1-\sin \left(\frac{\phi_{i j}}{2}\right)}{1+\sin \left(\frac{\phi_{i j}}{2}\right)} \\
T_{1 i j} & =\frac{1}{\omega_{i j} \sqrt{\alpha_{i j}}}, \quad T_{2 i j}=\alpha_{i j} T_{1 i j}, \quad L_{i j}^{1}(s)=\frac{\left(1+s T_{1 i j}\right)^{2}}{\left(1+s T_{2 i j}\right)^{2}}
\end{aligned}
$$

where $A_{i j 0}$ is the residue derived from separating the pair of poles, shown in (2), and all other parameters are obtained from Table 2. Here, index $i=1,2$ represents the dominant mode, while index $j=3$, 4 represents the phase angles fed back.

- Design the simple low-pass filters

$$
L_{i j}^{2}(s)=\frac{1}{1+s T_{i j}}, \quad T_{i j}=\frac{2 \pi}{\frac{4}{3} \omega_{i j 0}}, \quad i=(1,2), \quad j=(3,4) .
$$

- Design the high-pass washout filters

$$
L_{i j}^{3}(s)=\frac{s k_{w} T_{w}}{1+s T_{w}}, \quad \forall(i, j),
$$

where $k_{w}$ is the filter gain, and $T_{w}$ is the filter time constant. For our designs, we set $k_{w}=0.05, T_{w}=1$.

- Combine the subsystem transfer functions into a cascaded controller:

$$
L_{i j}:=L_{i j}^{1} L_{i j}^{2} L_{i j}^{3}, \quad i=(1,2), \quad j=(3,4) .
$$

The designs for these four sets of transfer functions are obtained as:

$$
\begin{aligned}
& L_{13}(s)=\left(\frac{1+1.935 s}{1+0.332 s}\right)^{2}\left(\frac{1}{1+4.125 s}\right)\left(\frac{0.05 s}{1+s}\right) \\
& L_{23}(s)=\left(\frac{1+1.262 s}{1+0.217 s}\right)^{2}\left(\frac{1}{1+2.625 s}\right)\left(\frac{0.05 s}{1+s}\right) \\
& L_{14}(s)=\left(\frac{1+1.889 s}{1+0.324 s}\right)^{2}\left(\frac{1}{1+3.836 s}\right)\left(\frac{0.05 s}{1+s}\right) \\
& L_{24}(s)=\left(\frac{1+1.378 s}{1+0.236 s}\right)^{2}\left(\frac{1}{1+2.802 s}\right)\left(\frac{0.05 s}{1+s}\right)
\end{aligned}
$$

- Finally, the controller input is computed as

$$
I(s)=\left(L_{13}(s)+L_{23}(s)\right)\left(\theta_{3}(s)-\theta_{4}(s)\right)+\left(L_{14}(s)+L_{24}(s)\right)\left(\theta_{4}(s)-\theta_{5}(s)\right)
$$


which is consistent with the block diagram shown in Figure 3. This input signal is added to the nominal input signal of the PI controller of the SVC. If needed, these controller parameters can be updated at regular intervals to compensate for changes in the actual system model.

\section{Simulation Results}

The designed controller in Section 5 is implemented in RSCAD using phase angle 3 as the feedback variable for the first case. For the second case, the angle 4 is also included, as in (13). These angles are chosen due to their low variance, shown in Table 1. The model is then disturbed with an eight-cycle, three-phase line-to-ground fault pilot bus 3 . The resulting phase angle disturbance is recorded for three cases. The first case uses the SVC with no supplementary controller. The second case uses the supplementary controller designed in Section 5. The third case uses supplementary controllers with two phase angle inputs from the model rather than just one, gathering data from areas 3, 4, and 5. The phase angles are plotted against one another in Figure 5. Table 3 and 4 display the damping values for each mode of each closed-loop phase angle when we implement the two controllers.
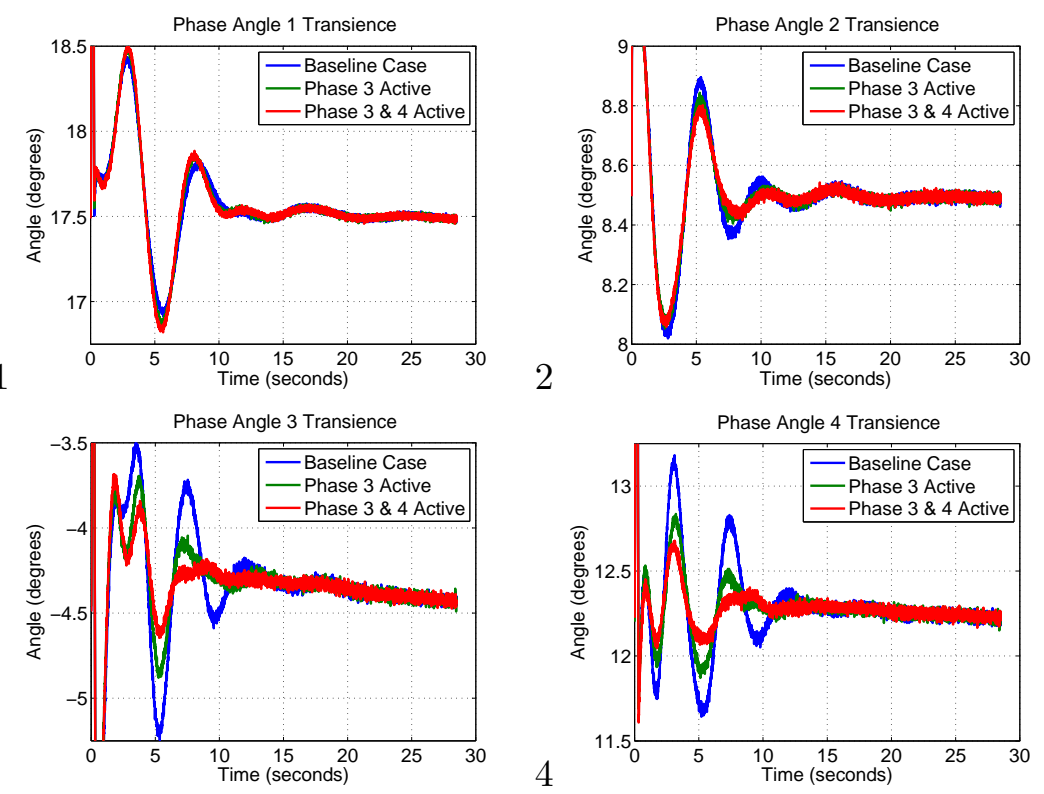

Figure 5: Closed-Loop Phase Angle Responses 
Table 3: Changes in Damping for Mode 1

\begin{tabular}{|c|c|c|c|}
\hline $\begin{array}{c}\text { Phase } \\
\text { Angle }\end{array}$ & $\begin{array}{c}\text { Mode 1 Damping } \\
\text { Baseline }\end{array}$ & $\begin{array}{c}\text { Mode 1 Damping } \\
\text { Control 1 }\end{array}$ & $\begin{array}{c}\text { Mode 1 Damping } \\
\text { Control 2 }\end{array}$ \\
\hline 1 & 0.3653 & 0.3311 & 0.3426 \\
2 & 0.6006 & 0.7816 & 1.0000 \\
3 & 0.2166 & 0.3747 & $0.2856^{*}$ \\
4 & 0.2774 & 0.3001 & 0.4143 \\
\hline
\end{tabular}

Table 4: Changes in Damping for Mode 2

\begin{tabular}{|c|c|c|c|}
\hline $\begin{array}{c}\text { Phase } \\
\text { Angle }\end{array}$ & $\begin{array}{c}\text { Mode 2 Damping } \\
\text { Baseline }\end{array}$ & $\begin{array}{c}\text { Mode 2 Damping } \\
\text { Control 1 }\end{array}$ & $\begin{array}{c}\text { Mode 2 Damping } \\
\text { Control 2 }\end{array}$ \\
\hline 1 & 0.1923 & 0.1616 & 0.1674 \\
2 & 0.2213 & 0.2762 & 0.2840 \\
3 & 0.2763 & 0.3589 & $0.2856^{*}$ \\
4 & 0.1472 & 0.2151 & 0.4029 \\
\hline
\end{tabular}

For phase angle 3 the ERA decomposition did not show the same frequencies as observed in the previous two tests as a result of which the closed-loop damping was not directly comparable with those for the other cases. Only one mode at a frequency between those observed in the previous two cases was computed, and the damping of this new mode was recorded in Tables 3 and 4. Figure 5, however, clearly indicates that a similar improvement is seen in the second test over that of the first test, and then over the baseline model with no supplementary controller. Tables 3 and 4 display the changes in damping for the two dominant slow modes after the supplementary controller was implemented, and expanded to have more than one relative phase angle for feedback. With the exception of the most geographically distant phase, all modes became significantly more damped. The angles between areas 3 and 4 , and between areas 4 and 5 show substantial improvement in damping. The damping of the angle between areas 2 and 3 is modestly improved. The angle between areas 1 and 2 is relatively less damped. Overall, however, the controller using two relative phase angles exhibits a significantly high level of damping that outweighs the degradation of dynamic performance seen from local or decentralized control in Fig. 2, This clearly illustrates the benefit of using the wide-area supplementary control. 


\section{Computation of SVC Capacity for Wide-Area Control}

In this section we study how much capacity is demanded from the SVC during transience when the wide-area control loop is executed. To answer this question, we perform three sets of tests, namely by varying fault duration, SVC droop, and level of wind penetration in area 4 . The model is faulted, and the resulting phase angles across all four inter-area transfer paths are recorded in RSCAD. The reactive power injection from the SVC is also recorded during each test to evaluate its contribution on the phase angle dynamics. The data collected from the SVC is filtered through a bandpass filter with passband between $w_{l}=2 \pi * 0.01 \mathrm{rad} / \mathrm{s}$ and $w_{u}=2 \pi * 2.5 \mathrm{rad} / \mathrm{s}$ in order to remove much of the higher frequency noise due to various power electronic converter dynamics coupled with the wind generator. Figure 6 shows an example of a set of collected data from the SVC, both pre and post-filtering. The maximum and minimum reactive power outputs delivered by the SVC during transience occur at $t_{\max }=1.45 \mathrm{~s}$ and $t_{\min }=1.70$ $\mathrm{s}$, respectively. These time points will be used in the subsequent sections to determine the capacity requirement of the SVC.

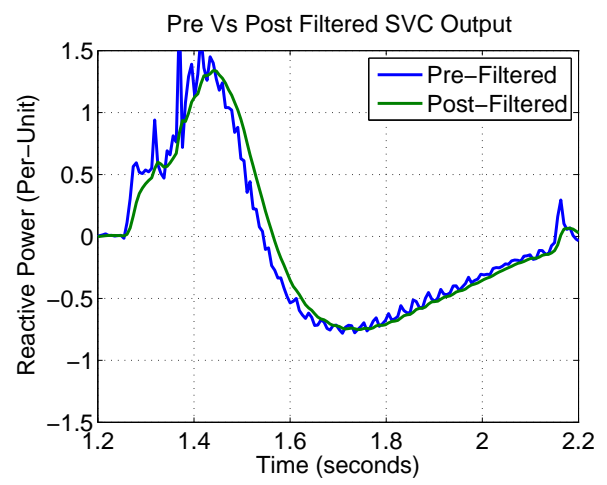

Figure 6: SVC Reactive Power Output (Pre and Post-Filtered)

\subsection{Test 1 - Variation in Fault Duration}

The first test involves testing the WECC model with faults of differing duration. These faults were chosen three phase line-to-ground faults at the pilot bus 3, which is, roughly speaking, the midpoint of the interconnection. The duration of fault was swept from one cycle to six cycles. Faults of duration longer than six cycles yielded results that caused the SVC to clip. During this test, the other two variables of interest, SVC droop and wind penetration in area 4 , were held at nominal values of $4 \%$ and $27 \%$, 
respectively. As seen in the first two plots of Figure 7, one can observe a linear relationship between fault duration and the maximum and minimum reactive power injections from the SVC during transience.

\subsection{Test 2 - Variation in SVC Droop}

The second test involves testing the WECC model with different droop values for the SVC. This droop has no dependence on the wide-area controller, and is present in a standard SVC configuration [6]. Droop was varied from $0 \%$ to higher values of $9 \%$, in increments of $1 \%$. The other two variables of interest, fault duration and wind penetration in area 4, were held at 4 cycles and $27 \%$, respectively. As seen in the second two plots of Figure 7. one can observe a weak linear relationship between SVC droop and the SVC maximum and minimum reactive power injections during transience. Voltage has relatively smaller effect on the wide-area controller. Any variation in SVC behavior is due to the effect that droop has on the local voltage response.

\subsection{Test 3 - Variation in Wind Penetration}

The third test involves testing the WECC model with varying levels of wind penetration at pilot bus 4 . Wind penetration was ranged from $0 \%$ to $54 \%$, and machine inertia was correspondingly subtracted from the aggregate machine 4. During this test, the other two variables of interest, SVC droop and fault duration, were held at $4 \%$ and 4 cycles, respectively. As seen in the two last plots in Figure 7, there is little to almost no correlation between wind penetration in area 4 and the intensity of the swing of the reactive power injection from the SVC.

\subsection{Test Results}

Next, a linear regression is fitted to the data obtained from these tests, thereby generating a heuristic equation predicting the SVC reactive power necessary (in both the positive and negative directions) to ensure that clipping does not occur during transience while the wide-area control loop is active. This predictive model is obtained as

$$
\begin{aligned}
& C_{\max }=\alpha_{1} * W+\beta_{1} * F+\gamma_{1} * D+\xi_{1} \\
& C_{\text {min }}=\alpha_{2} * W+\beta_{2} * F+\gamma_{2} * D+\xi_{2}
\end{aligned}
$$

where, $\alpha_{1}=-0.0015, \alpha_{2}=-0.0002, \beta_{1}=0.2567, \beta_{2}=-0.1535, \gamma_{1}=$ $-0.0118, \gamma_{2}=0.0177, \xi_{1}=0.1868, \xi_{2}=-0.1498, W$ is the percentage of wind power penetration at pilot bus $4, F$ is fault duration given in cycles, 

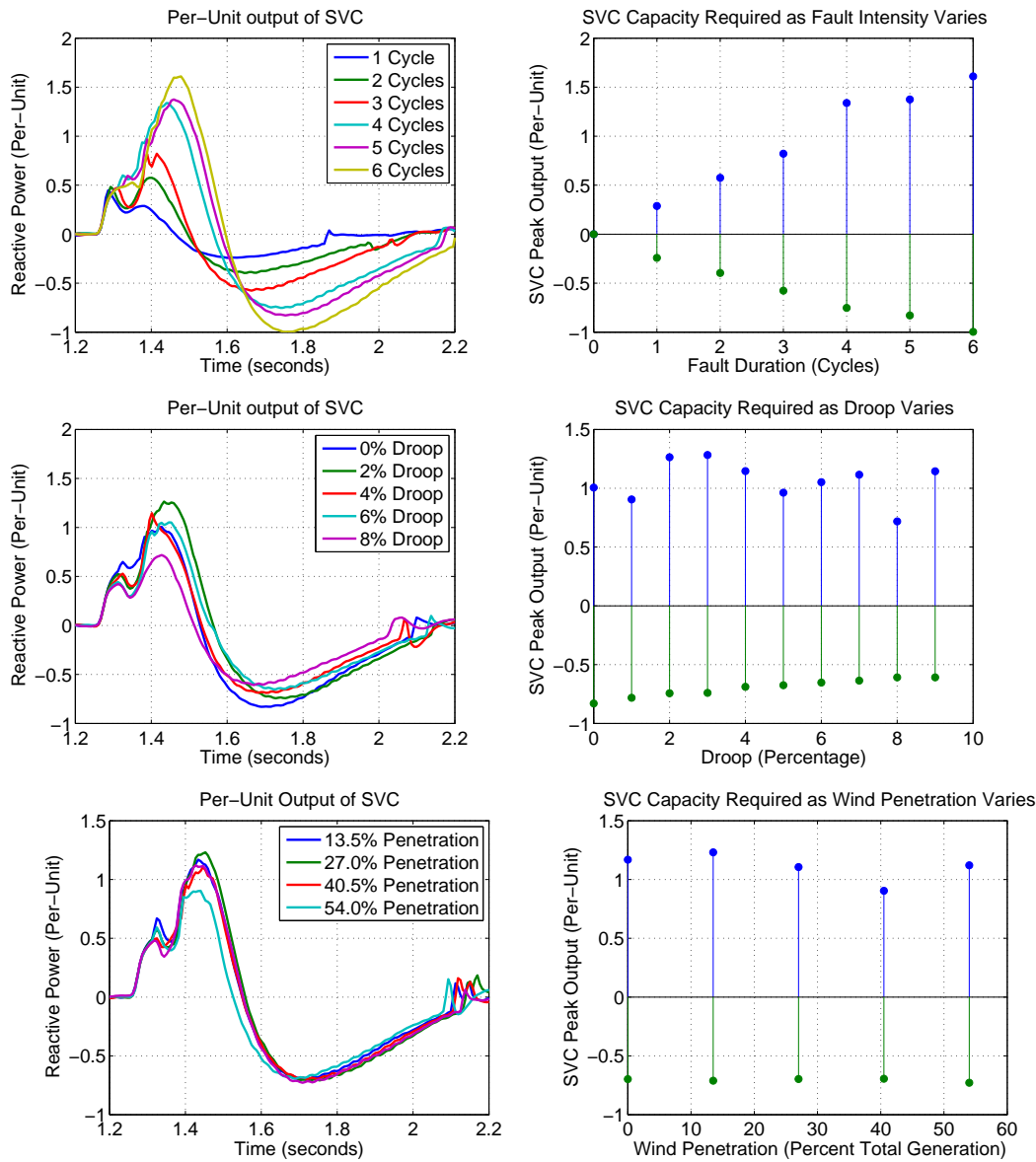

Figure 7: Simulation Results for Computing SVC Capacity Requirements 
$D$ is the SVC droop, $C_{\max }$ is the amount of VAr overhead needed by the SVC for not clipping, and $C_{\text {min }}$ is the amount of VAr underhead needed by the SVC for not clipping. These equations are verified by collecting many additional sets of tests conducted at various critical values of the model parameter given in [1. All of these tests matched the identified model with error less than $10^{-5}$.

\section{Validation of the Controller Against Varying Power Flows}

In all the experiments carried out so far, the pre-disturbance equilibrium power flow across each line of the equivalent model has been held constant whereas other variables such as SVC parameters, control gains, wind penetration, etc. have been varied. We next perform a set of tests to validate the robustness of our SVC controller against changing operating points, or equivalently against changing load-generation setpoints that result in different steady-state megawatts across the different transfer paths.

Three test cases are considered. The load reference sliders in the model were altered to create several different power flow scenarios. Line impedance, intra-area impedance, machine inertia, machine damping, and wind penetration were maintained constant at their nominal identified values. The only quantities varied were inter-area line power flows. The schematic for this power flow are shown in the top-left of Figures 8, 9, and 10. Levels of stress in each machine and along each transmission line are color-coded. Absolute steady-state angles can be seen for each case in the individual plots for each figure. Each test case contains two sets of data. One set of data, plotted in green, was collected by faulting the model but with the wide-area control disabled. Note that the SVC is still enabled in these cases, but is simply operating as a voltage regulator with no supplementary control. The other set of data plotted against the first set contains the case where the model was faulted, but now with the wide-area controller online. The per-unit reactive power output of the SVC is plotted for each case, together with the four phase angle differences between each pair of the neighboring areas.

\subsubsection{Case 1}

Case 1 is the baseline case, where the transfer flows are maintained at their nominal values following [1]. The results, plotted in Fig. 8, for this test case are similar to that of Fig. 5. The only difference between the two test cases is the length of the fault. Nonetheless, one can see significant improvements in the transient responses of phase angles 3 and 4 when the wide-area controller is implemented. 


\subsubsection{Case 2}

Case 2 is designed to stress the southern portion of the WECC, containing areas 4 and 5. In Figure 9, one can see the machines along the bottom are stressed, and the angles across those transmission lines are larger than the nominal case. The third phase angle has switched direction, indicating power is now flowing from area 4 to area 3 in contrast to the nominal case. When the wide-area controller is switched off, the model is immediately unstable after fault. When the controller is online, however, the model is not only stable but significantly damped.

\subsubsection{Case 3}

Case 3 is designed to stress the northern and central portions of the WECC, containing areas 1, 2, and 3. In Figure 10, one can see that the machines along the northern and central parts are stressed, and the angles across the corresponding transmission lines are larger than the nominal case. Power now flows in the opposite direction for both areas compared to the nominal case. The model is marginally unstable without the wide-area controller. The machines distant from the SVC are first to become asynchronous with the grid under this operating condition. When the wide-area controller is switched on, the closed-loop angle responses become stable.

\section{Conclusion}

In this paper we developed a wide-area damping control design via SVC supplementary control using a dynamic five-area equivalent model of the WECC system as a baseline. We showed how the small-signal closed-loop dynamic performance of the inter-machine swing oscillations in the reducedorder system, improve with the SVC control. These oscillation, in turn, correspond to inter-area oscillations in the actual full-order network. Since the identities of the pilot buses in the two models are identical, and the SVC is a shunt controller, hence the controller can be easily implemented in the actual WECC system. Simulation results reveal the effectiveness of the wide-area controller in improving dynamic performance, especially when wind penetration is added in area 4 . Our future work will include extension of this design to multiple SVC controllers over a distributed communication network, and test the transient performance of the closed-loop system with respect to communication delays and data loss. 

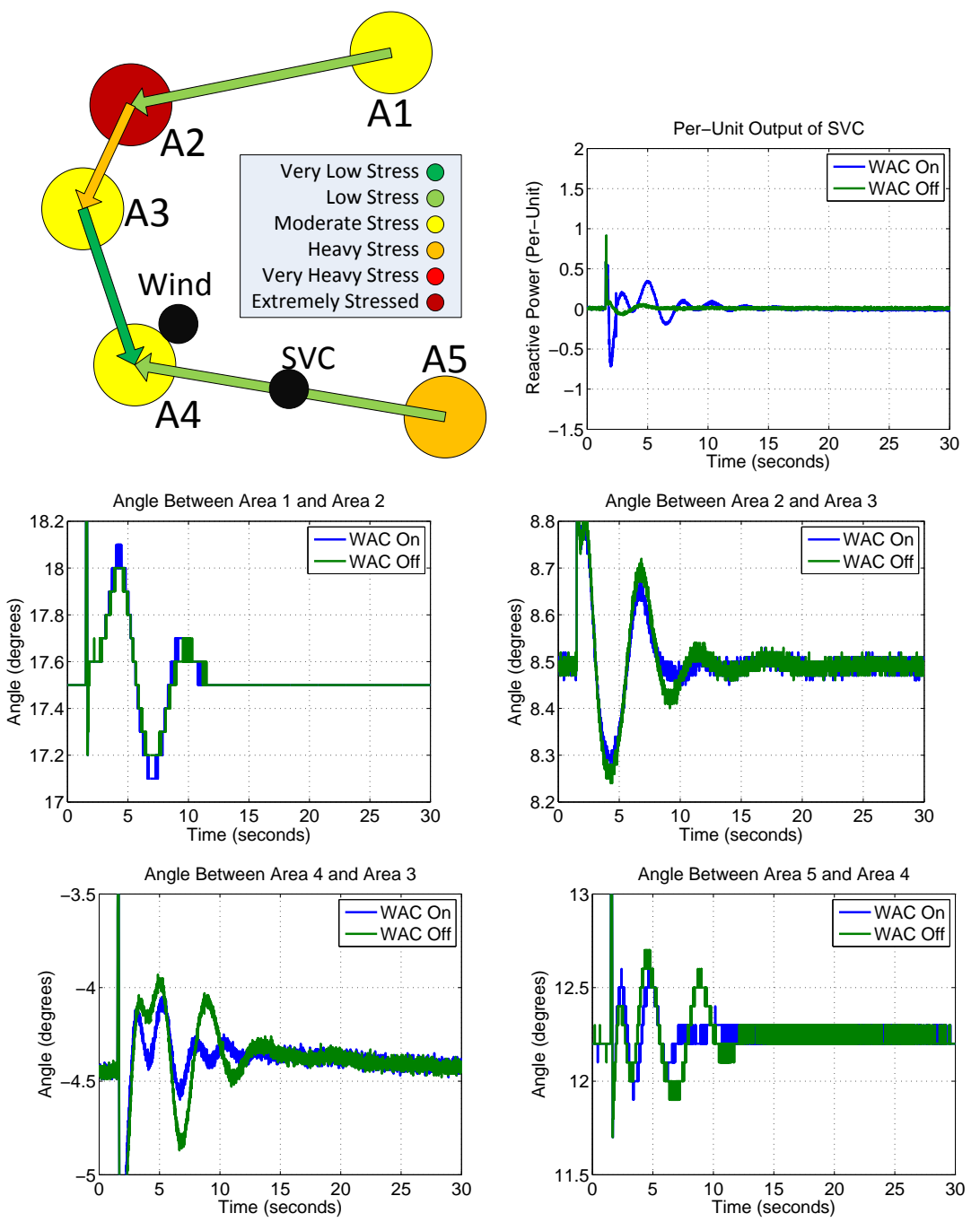

Figure 8: Closed-Loop Responses for Case 1 

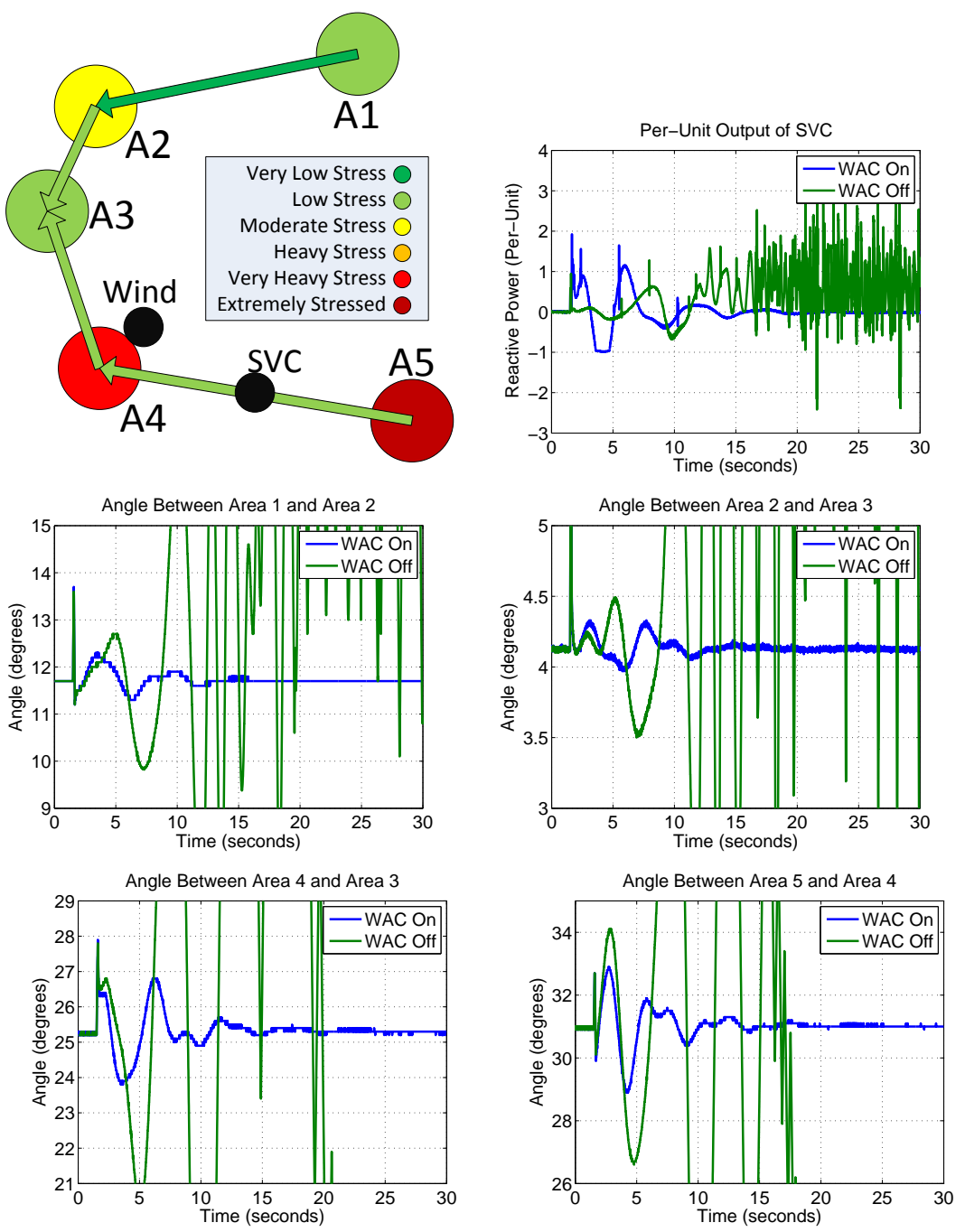

Figure 9: Closed-Loop Responses for Case 2 

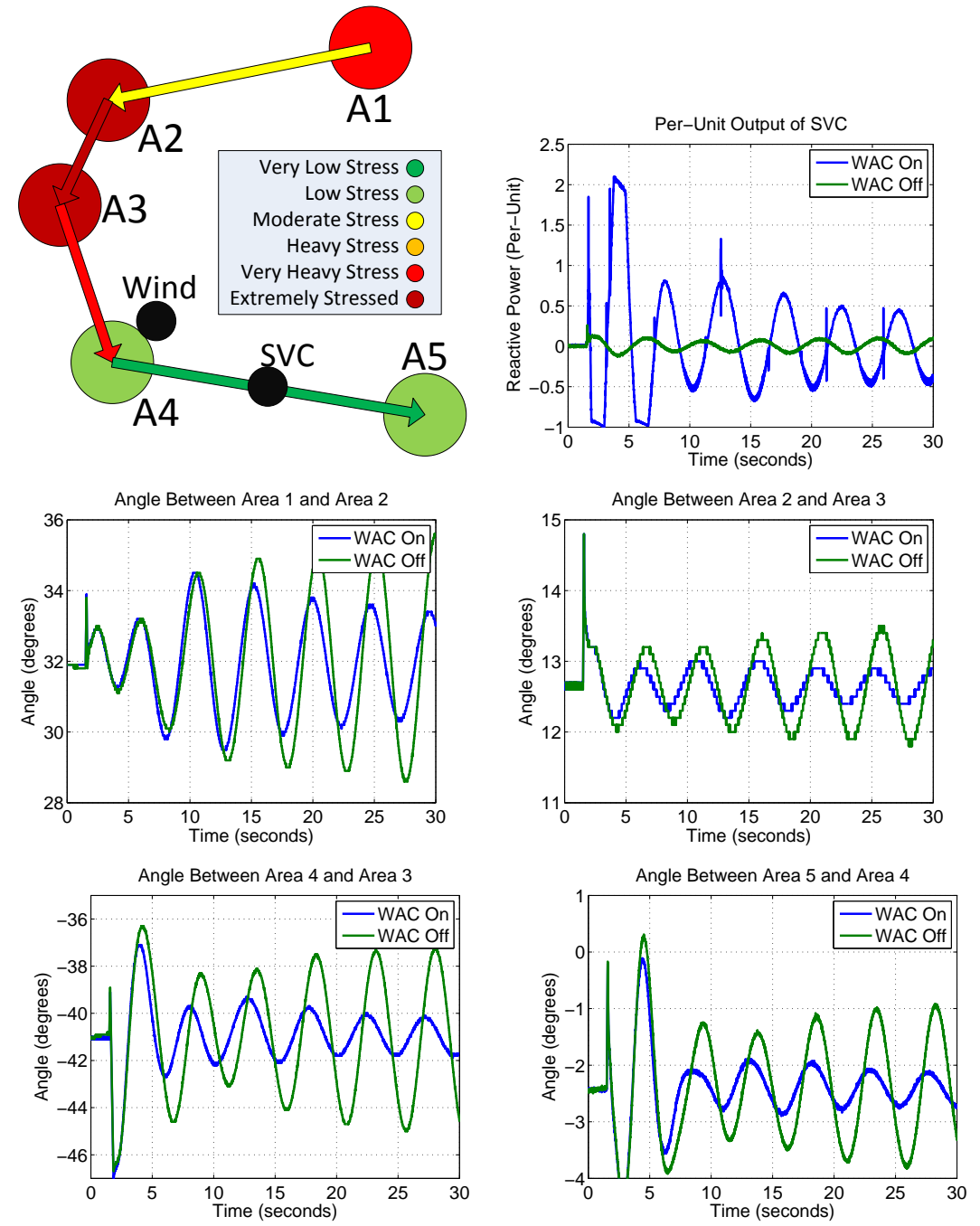

Figure 10: Closed-Loop Responses for Case 3 


\section{References}

[1] G. Chavan, M. Weiss, A. Chakrabortty, S. Bhattacharya, A. Salazar and F. Ashrafi, "Wide-Area Monitoring and Control of WECC Transfer Paths Using Real-Time Digital Simulations," Technical Report, NC State University, 2013 (available online: http://people.engr.ncsu.edu/achakra2/scereport.pdf)

[2] P. Kokotovic, J. Allemong, J. Winkelman, J. H Chow, "Singular perturbation and iterative separation of time scales," Automatica, vol. 16 (1), pp. 23-33, 1980.

[3] J. Ballance, B. Bhargava, and G. D. Rodriguez, "Use of Synchronized Phasor Measurement System for Enhancing AC-DC Power System Transmission Reliability and Capability," Eastern Interconnection Phasor Project, (EIPP) Meeting, Sep. 2004.

[4] A. G. Phadke and J. S. Thorp, Synchronized Phasor Measurements and Their Applications, New York, Springer, 2008.

[5] A. Chakrabortty, "Wide-Area Control of Large Power Systems Using Dynamic Clustering and TCSC-Based Redesigns," IEEE Transactions on Smart Grid, vol. 3(3), Sep. 2012.

[6] N. G. Hingorani and L. Gyugyi, Understanding FACTS: Concepts and Technology of Flexible AC Transmission Systems, Wiley, 1999.

[7] P. Pourbeik, A. Bostrom and B. Ray, "Modeling and Application Studies for a Modern Static VAr System Installation," IEEE Transactions on Power Delivery, vol. 21, no. 1, pp. 368-377, Jan. 2006

[8] J. H Chow and K. W Cheung, "A toolbox for power system dynamics and control engineering education and research," Power Systems, IEEE Transactions on, vol. 7 (4), pp. 1559-1564, 1992.

[9] C. W. Taylor and V. Venkatasubramanian, "Wide-Area Stability and Voltage Control," in Proc. VII Symposium of Specialists in Electric Operational and Expansion Planning SEPOPE, May 2000.

[10] Y. Chang, Z. Xu, G. Cheng, and J. Xie, "A novel SVC supplementary controller based on wide area signals," IEEE PES General Meeting, 2006. 